\title{
Modified Transport-of-Intensity Approach for Mapping In-situ Magnetic Induction
}

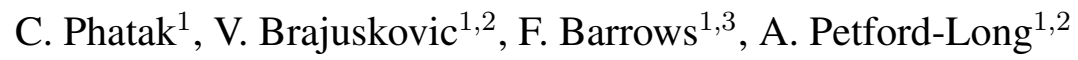 \\ ${ }^{1}$ Materials Science Division, Argonne National Laboratory, Lemont, IL 60439, USA \\ ${ }^{2}$ Department of Materials Science and Engineering, Northwestern University, Evanston, IL 60208, \\ USA \\ ${ }^{3}$ Applied Physics Program, Northwestern University, Evanston, IL 60208, USA
}

Lorentz tranmission electron microscopy (LTEM) is ideally suited for quantitative analysis of magnetic domains at the nanometer length scale. The ability to study both the microstructure and magnetic domain structure simultaneously in functional materials allows for direct understanding of the fundamental role of inhomogeneities in microstructure as well as the effect of shape and size of nanostructures on the magnetic domain behavior. Quantitative information about the magnetization is contained in the phase shift of electrons as given by the Aharonov-Bohm phase shift.[1] There are several ways to recover this phase shift such as using the transport-of-intensity equation (TIE) formalism or off-axis electron holography. The phase shift consists of electrostatic and magnetic components, which are related to the projection of the electrostatic potential and the magnetic vector potential respectively. To study the magnetization state of an object, it is necessary to separate these phase shifts, which is achieved using time reversal symmetry i.e., retrieveing the phase shift with the sample in upright orientation and $180^{\circ}$ flipped over orientation and then substracting one from the other. This method is not suitable for in-situ experiments, where the sample flip at every stage of the in-situ experiment is not practical. In this work, we present a modified version of the TIE formalism to retrieve the magnetic phase shift during an in-situ experiment.

In the TIE formalism, the total phase shift is recovered by numerically solving the equation [2]: $\nabla \cdot\left[I_{0} \nabla \varphi\right]=-k \partial_{z} I$, where $k=2 \pi / \lambda, I_{0}$ is the in-focus image intensity, and $\partial_{z} I$ is the intensity derivative along the direction of propagation of electrons. The intensity derivative is typically determined from the through-focus series of images using the central difference method. The individual phase shifts are then recovered by subtracting the two phase images. However, in our previous work, we have shown that due to the linear nature of the equation and relationship between phase shifts, the phase separation can be performed by subtracting the images directly. [3] Here we extend this to the images acquired during an in-situ experiment such as in-situ magnetizing or thermal annealing, where $\left[I_{-}^{n+}, I_{0}^{n+}, I_{+}^{n+}\right]$ represents a through-focus series of images acquired at the $n^{t h}$ step of the experiment. We also assume that before the start of the experiment, we have recorded a through-focus series of images with the sample in upright orientation, $\left[I_{-}^{0+}, I_{0}^{0+}, I_{+}^{0+}\right]$, and the $180^{\circ}$ flipped over orientation, $\left[I_{-}^{0-}, I_{0}^{0-}, I_{+}^{0-}\right]$. Here the superscript \pm represents the upright or flipped over orientation, and the subscript \pm represents the over-focus or under-focus condition respectively. Under the assumption that the electrostatic potential and the in-focus image intensity does not change during the in-situ experiment, we can directly solve for the magnetic phase shift at the $n^{\text {th }}$ step as:

$$
\nabla \cdot\left(I_{0}^{n} \nabla \varphi_{m}^{n}\right)=-\frac{k}{2 \Delta f}\left[I_{+}^{n+}-I_{-}^{n+}-\frac{1}{2}\left(I_{+}^{0+}-I_{-}^{0+}+I_{+}^{0-}-I_{-}^{0-}\right)\right]
$$

We have applied this new approach to magnetic phase shift reconstruction of simulated in-situ mag- 
netizing of coupled NiFe discs. Figure 1(a) shows the under-focus LTEM image, (b) and (c) show the recovered magnetic and electrostatic phase shift at the initial state. These phase shifts were recovered using both the images of upright and flipped upside down orientation. Figure 1(d) shows the underfocus LTEM image in upright orientation at simulated applied field of $H=160$ Oe, and (e) shows the recovered magnetic phase shift using our modified TIE approach. Figure 1(f) shows a comparison of the recovered magnetic phase shift and the theoretical phase shift along the dashed line shown in Figure 1(e). It can be seen that the magnetic phase shift is almost exactly recovered using our modified approach. The advantage of this approach is that it offers reduced noise in the reconstructed magnetic phase images as compared to that obtained using subtraction of phase images directly. We will also present application of this method for experimental in-situ magnetizing as well as thermal annealing experiments to show the magnetic domain mapping obtained at various stage during the experiment.

\section{References:}

[1] Y. Aharonov and D. Bohm, Phys. Rev., 115(3), 485 (1959).

[2] M. Teague, J. Opt. Soc. Amer., 72(9), 1199 (1982).

[3] E. Humphrey, C. Phatak, A.K. Petford-Long, M. De Graef, Ultramicroscopy, 139, 5 (2014).

[4] This work was supported by U.S. Department of Energy (DOE), Office of Science, Basic Energy Sciences, Materials Sciences and Engineering Division.
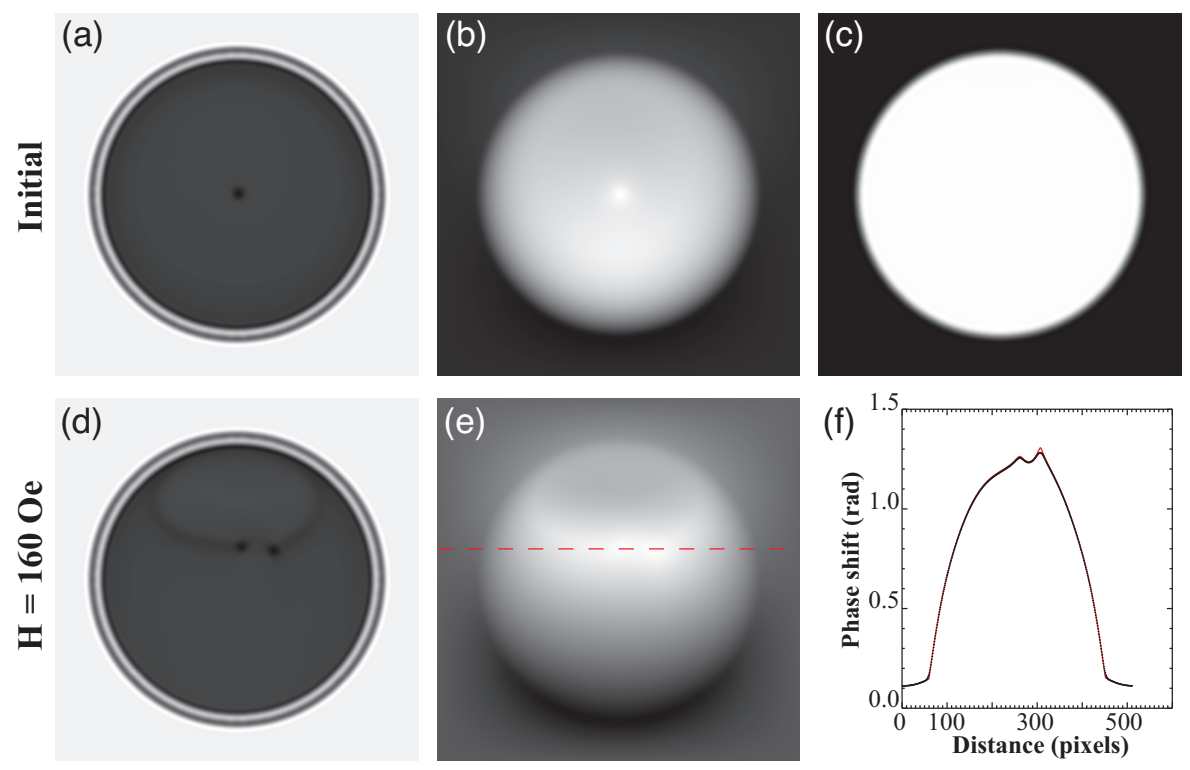

Figure 1: (a) shows the under-focus LTEM image, (b) and (c) show the recovered magnetic and electrostatic phase shift at the initial state using upright and flipped upside image series. (d) shows the under-focus LTEM image in upright orientation, and (e) shows the recovered magnetic phase shift using the modified TIE approach at simulated applied field of $\mathrm{H}=160 \mathrm{Oe}$. (f) shows the comparison of the recovered magnetic phase shift (black) and the theoretical magnetic phase shift (red) along the dashed line in (e). 